Audiology

\title{
Objective assessment of subjective tinnitus through contralateral suppression of otoacoustic emissions by white noise: effects of frequency, gender, tinnitus bilaterality and age
}

\author{
Valutazione oggettiva dell'acufene soggettivo attraverso la soppressione \\ controlaterale delle otoemissioni acustiche effettuata con rumore bianco: \\ effetti di genere ed età, di frequenza e bilateralità dell'acufene
}

M. RIGA' ${ }^{1}$, A. KOMIS ${ }^{2}$, P. MARAGOUDAKIS ${ }^{3}$, G. KORRES ${ }^{3}$, E. FEREKIDIS ${ }^{2}$, V. DANIELIDES ${ }^{1}$

${ }^{1}$ Democritus University of Thrace, Alexandroupolis, Greece; ${ }^{2}$ University ENT Dept, Hippoktation General Hospital of Athens, Greece; ${ }^{3}$ University ENT Dept, Attikon University Hospital, Athens, Greece

\section{SUMMARY}

Accumulating evidence seems to support an association between tinnitus and medial olivocochlear bundle (MOCB) dysfunction. Most studies use patient/control comparisons to support this correlation. The aim of this study was to investigate the hypothesis in a substantially different way and evaluate the roles of gender, age, frequency and tinnitus bilaterality as possible confounding factors. The population consisted of 78 normal hearing patients with chronic tinnitus, 28 normal hearing controls, 19 presbycousic tinnitus patients and 13 presbycousic controls ( $\mathrm{n}=276$ ears). Mean suppression amplitudes of transient evoked otoacoustic emissions (TEOAEs) and distortion product OAEs (DPOAEs) by contralateral white noise (50 dB SPL) were computed. Mean suppression values $<1 \mathrm{~dB}$ SPL or $<2 \mathrm{~dB}$ SPL were validated as positive test results. Overall suppression (OS) values $<1 \mathrm{~dB}$ SPL were qualified as a diagnostic test of moderate positive predictive value for both DPOAEs and TEOAEs, while OS values $<2 \mathrm{~dB}$ SPL were found to be of large negative predictive value for DPOAEs and moderate for TEOAEs. Mean suppression values (for all frequencies, OS) are of higher diagnostic value than suppression values corresponding to either lower (1-2 kHz) or higher frequencies (2.8-4 kHz for TEOAEs and 2.8-6 kHz for DPOAEs). After excluding patients with unilateral tinnitus from the analysis, correlations were found to be stronger. Useful correlations were also attributed for all age groups $<61$ years. In females, OAE suppression seems to have a stronger positive predictive value, while in males it seems to have a stronger negative predictive value. OAE-based assays of MOCB function as an objective diagnostic tool for subjective tinnitus might deserve further investigation. Tinnitus uni- or bi-laterality is a confounding factor, which probably confirms the observation that defective function of the MOCB usually applies to the contralateral ear as well. Gender is an additional confounding factor, while correlations can be verified for all age groups $<61$ years old.

KEY WORDS: Tinnitus • Diagnosis • Specificity • Sensitivity • Contralateral suppression of otoacoustic emissions

\section{RIASSUNTO}

Evidenze sempre più numerose sembrano supportare l'esistenza di un'associazione tra acufene e disfunzione del fascio olivococleare mediale (FOCM). Svariati studi utilizzano il confronto paziente/controllo per sostenere questa correlazione. L'obiettivo di questo studio è stato quello di indagare la suddetta ipotesi in una maniera differente e valutare il ruolo del genere, dell'età, della frequenza e bi-lateralità dell'acufene come fattori confondenti. La popolazione comprendeva 78 pazienti normoudenti con acufene cronico, 28 controllo normoudenti, 19 pazienti presbiacusici con acufene e 13 presbiacusici di controllo (per un totale di 276 orecchie). È stato calcolato il valore medio di soppressione dell'ampiezza delle otoemissioni evocate da transienti (TEOAEs) e dei prodotti di distorsione (DPOAEs) utilizzando un rumore bianco controlaterale di $50 \mathrm{~dB}$. Valori medi della soppressione inferiori a $1 \mathrm{~dB}$ SPL o $2 \mathrm{~dB}$ SPL sono stati validati come positivi. Valori di soppressione inferiori a $1 \mathrm{~dB} S P L$, sia per le TEOAEs sia per le DPOAEs, sono stati considerati valori di moderato valore predittivo positivo, mentre valori di soppressione inferiori a $2 \mathrm{~dB}$ SPL sono stati considerati valori di alto valore predittivo negativo per le DPOAEs, e di moderato valore predittivo negativo per le TEOAEs. I valori di soppressione medi, ovvero per tutte le frequenze, sono di più alto valore diagnostico rispetto a quelli specifici per le basse frequenze (1-2 kHz) e per le alte frequenze (2,8-4 kHz per le TEOAEs e 2,8-6 kHz per le DPOAEs). Dopo aver escluso i pazienti con acufene monolaterale, le correlazioni effettuate si sono dimostrate più forti. Valide correlazioni sono state effettuate per tutti $i$ gruppi di età inferiore a 61 anni. Inoltre, sembra che la soppressione per le TEOAEs abbia nelle donne un valore predittivo positivo maggiore, mentre nei maschi un valore predittivo negativo maggiore. Lo studio della funzionalità del fascio olivococleare mediale mediante otoemissioni, in qualità di test di valutazione oggettiva degli acufeni, meriterebbe ulteriori studi. La bi-lateralità dell'acufene è un fattore confondente, che conferma, probabilmente, la constatazione che la disfunzione del fascio olivococleare mediale riguarda solitamente anche l'orecchio controlaterale. Il sesso è un altro fattore confondente, mentre le varie correlazioni possono essere verificate in tutti i gruppi di età inferiori a 61 anni.

PAROLE CHIAVE: Acufene $\bullet$ Diagnosi $\bullet$ Sensibilità $\bullet$ Specificità $\bullet$ Soppressione controlaterale di otoemissioni

Acta Otorhinolaryngol Ital 2018;38:131-137 


\section{Introduction}

Tinnitus represents an important challenge for audiology and neurootology research in a number of ways. First, the underlying pathophysiology remains obscure. Patients reporting tinnitus have been found to exhibit defective medial olivocochlear bundle (MOCB) function, when assessed through contralateral suppression of otoacoustic emissions (OAEs) by acoustic stimuli in a number of studies ${ }^{1-8}$. However, some researchers, who adopted substantially different methods of analysing and evaluating their results, have failed to establish a connection between MOCB dysfunction and tinnitus generation ${ }^{910}$. A review of the relevant literature ${ }^{11}$ concluded that reduction of the neural efferent control of the cochlear amplifier seems to represent one of the possible pathophysiological abnormalities that may be related to tinnitus in normal hearing patients. Second, there is no objective examination that can measure or simply verify the existence of tinnitus. Diagnosis is based solely on the patient's report, thus raising reasonable doubts in cases where psychological issues, or any potential insurance, professional, or pension benefits are involved.

The aim of this study was to assess contralateral suppression of OAEs by acoustic stimulus as a potential tool for an objective investigation of tinnitus and investigate the roles of gender, age and tinnitus bi- or uni-laterality as possible confounding factors. Another aim of the study was to investigate any frequency-related differences that may reveal an association between high-frequency tinnitus and suppression malfunction at higher frequencies. Relevant implications regard both diagnostic and therapeutic assessment of tinnitus through focal or pharmacological manipulation of the olivocochlear system ${ }^{12}$.

\section{Materials and methods}

The population of this study consisted of 110 right handed adults with chronic (more than 3 months) subjective tinnitus of high frequency and 28 controls ( $n=276$ ears), all with measurable transient evoked OAEs (TEOAEs) and/or distortion product OAEs (DPOAEs) in at least two frequencies. When no measurable OAEs were present no value was registered for the respective frequency/ies. The population included 78 normal hearing tinnitus patients (mean age \pm standard deviation; $46 \pm 14$ years, 45 females and 33 males), 28 normal hearing controls (aged $42 \pm 13,15$ females and 13 males), 19 presbycousic subjects with bilateral tinnitus (aged $60 \pm 6,11$ females and 8 males) and 13 presbycousic controls (aged $60 \pm 6,7$ females and 6 males). Tinnitus was lateralised to the right in 15 , to the left in 26 and bilateral in 37 of normal hearing individuals. Cerebral dominance was considered as a potential confounding factor because of its presumed role in the lateralisation of the contralateral suppression of OAEs ${ }^{13}$. Normal hearing was determined according to the ISO 7029 norm specified for subject's age. Patients with retrocochlear tumours, middle ear pathologies or possible inner ear diseases known to be associated with tinnitus, such as possible Ménière's disease, otosclerosis and sudden hearing loss were excluded from the study. None of the subjects included reported a medical history of traumatic brain injury, or psychological or mood disorders. Transient evoked otoacoustic emissions (TEOAEs) and distortion product otoacoustic emissions (DPOAEs) were obtained using an ILO v6 apparatus (Otodynamics Ltd.) in a soundtreated booth at first in the absence and later in the presence of contralateral suppression (white noise of $50 \mathrm{~dB}$ SL presented continuously through headphones). TEOAEs were obtained by linear click stimuli of $60 \mathrm{~dB}$ SPL at 5 frequencies $(1,1.4,2,2.8$ and $4 \mathrm{kHz})$. DPOAEs were elicited by two tones of 65 and $55 \mathrm{~dB}$ SPL (L1 and L2 respectively) with $\mathrm{f} 2 / \mathrm{f} 1$ ratio being set at 1.22 at 6 frequencies $(1,1.4,2,2.8$, 4,5 and $6 \mathrm{kHz}$ ). Both TEOAEs and DPOAEs were considered valid when emission amplitude exceeded the noise by at least $6 \mathrm{~dB}$ SPL. The suppressor stimulus was contralateral white noise of $50 \mathrm{~dB}$ SPL, delivered by an Amplaid A321 Twin Channel (Amplifon, Milan, Italy) diagnostic audiometer. Contralateral suppression was calculated by subtracting the value of OAEs with contralateral white noise from the value of OAEs without contralateral noise. The study was approved by the Institutional Review Board and informed consent was obtained by all participants.

Suppression testing and data collection/transcription was done by one researcher, who was blinded to the conduction, design and cut-off criteria of this study. DPOAEs enhancement was substituted by zero values to avoid computational problems. Given the fact that there are no set normative values for either TEOAE or DPOAE suppression, the association between patient-reported tinnitus and low overall suppression values was estimated for 2 different criteria (less than 2 and $1 \mathrm{~dB}$ SPL). The selection of these criteria was based on a previous work ${ }^{14}$. The potentially different implication of lower and higher frequency suppression amplitudes in the final results was investigated by comparing the results obtained for overall suppression (OS) values to those calculated separately for lower and higher frequencies. The confounding role of tinnitus bilaterality was evaluated by analysing the results for the subgroup of patients with either bilateral or no tinnitus.

Descriptive statistics, used to summarise the findings, were analysed with SPSS software version 21.0 (SPSS, Inc., Chicago, USA). Likelihood ratios, sensitivity, specificity and positive and negative predictive values were calculated for ears with/without tinnitus $(\mathrm{n}=276)$. For $95 \%$ CIs on individ- 
ual proportions (sensitivity, specificity, positive/negative predictive values and positive/negative likelihood ratios), exact Clopper-Pearson confidence intervals were used. Statistical significance was attributed to two-sided $\mathrm{p}<0.05$.

\section{Results}

TEOAEs in at least two frequencies were recorded in all ears. The results on the sensitivity, specificity, positive and negative predictive values, positive and negative likelihood ratios for the six selected criteria used are summarised in Table I. Self-reporting of tinnitus was the "gold standard". DPOAEs and TEOAEs did not seem to differ significantly as diagnostic tools. Mean suppression values (for all frequencies, OS) are of higher diagnostic value than suppression values corresponding to either lower $(1-2 \mathrm{kHz})$ or higher frequencies (2.8-4 kHz for TEOAEs and 2.8-6 kHz for DPOAEs) (Table I).

Patients with unilateral tinnitus were excluded from the analysis presented in Table II. The diagnostic value of the test was therefore evaluated for patients with either bilateral tinnitus or no tinnitus. The results exhibited a trend toward better diagnostic potential than that calculated for the entire study population (Tables I, II). The results obtained after dividing the study population into four age groups are shown in Table III. Similar results were recorded for age groups 20-35, 36-50 and 51-60 years, while patients older than 61 years had inconclusive data.

Results by gender are presented in Table IV. Significant differences were present. In females, contralateral suppression of both TEOAEs and DPOAEs could be characterised as an often useful diagnostic tool for the presence of the disease when mean suppression values were less than $1 \mathrm{~dB}$ SPL, while DPOAEs was demonstrated to serve as an often useful tool that may indicate the absence of disease when mean suppression values were more than $2 \mathrm{~dB}$ SPL. In males, however, suppression recordings for the selected cut-off values seemed to be of diagnostic value only in terms of ruling out the presence of tinnitus.

\section{Discussion}

An important drawback in clinical practice and research on tinnitus is the absence of an objective examination that may provide any indication of its presence. The lack of diagnostic tools and the mystery around its pathogenesis

Table I. Results obtained for the cut-off criteria used in this study.

\begin{tabular}{|c|c|c|c|c|c|c|c|c|c|c|}
\hline \multicolumn{3}{|c|}{$\begin{array}{l}\text { Statistical test } \\
\text { cut-off criterion }\end{array}$} & \multirow{2}{*}{$\begin{array}{c}\text { Sensitivity } \\
95 \% \mathrm{Cl} \\
87 \% \\
81.05-92.25\end{array}$} & \multirow{2}{*}{$\begin{array}{c}\text { Specificity } \\
95 \% \mathrm{Cl} \\
67 \% \\
58.45-75.65\end{array}$} & \multirow{2}{*}{$\begin{array}{c}\text { PPV } \\
95 \% \mathrm{Cl} \\
77 \% \\
69.71-82.84\end{array}$} & \multirow{2}{*}{$\begin{array}{c}\text { NPV } \\
95 \% \text { Cl } \\
81 \% \\
72.45-88.4\end{array}$} & \multirow{2}{*}{$\begin{array}{c}\text { Positive LR } \\
\text { 95\% Cl } \\
2.69 \\
2.07-3.49\end{array}$} & \multirow{2}{*}{$\begin{array}{l}\text { Diagnostic } \\
\text { value } \\
\text { Small }\end{array}$} & \multirow{2}{*}{$\begin{array}{c}\text { Negative LR } \\
\text { 95\% Cl } \\
0.19 \\
0.12-0.29\end{array}$} & \multirow{2}{*}{$\begin{array}{c}\text { Diagnostic } \\
\text { value } \\
\text { Moderate }\end{array}$} \\
\hline OS & $\begin{array}{l}<2 \\
\mathrm{~dB} S P L\end{array}$ & TEOAEs & & & & & & & & \\
\hline & & DPOAES & $\begin{array}{c}95 \% \\
89.38-97.84\end{array}$ & $\begin{array}{c}64 \% \\
55.16-71.77\end{array}$ & $\begin{array}{c}71 \% \\
64.12-77.99\end{array}$ & $\begin{array}{c}93 \% \\
85.41-96.99\end{array}$ & $\begin{array}{c}2.61 \\
2.09-3.27\end{array}$ & Small & $\begin{array}{c}0.08 \\
0.04-0.17\end{array}$ & Large \\
\hline & $\begin{array}{l}<1 \\
\mathrm{~dB} S P L\end{array}$ & TEOAEs & $\begin{array}{c}54 \% \\
45.35-61.78\end{array}$ & $\begin{array}{c}91 \% \\
84.56-95.45\end{array}$ & $\begin{array}{c}88 \% \\
79.61-93.88\end{array}$ & $\begin{array}{c}62 \% \\
54.05-68.64\end{array}$ & $\begin{array}{c}6.00 \\
3.35-10.75\end{array}$ & Moderate & $\begin{array}{c}0.51 \\
0.43-0.61\end{array}$ & $\begin{array}{l}\text { Very } \\
\text { small }\end{array}$ \\
\hline & & DPOAES & $\begin{array}{c}75 \% \\
66.98-81.93\end{array}$ & $\begin{array}{c}88 \% \\
80.78-92.80\end{array}$ & $\begin{array}{c}87 \% \\
79.42-92.25\end{array}$ & $\begin{array}{c}77 \% \\
68.88-83.06\end{array}$ & $\begin{array}{c}6.09 \\
3.81-9.74\end{array}$ & Moderate & $\begin{array}{c}0.29 \\
0.21-0.38\end{array}$ & Small \\
\hline \multirow[t]{4}{*}{ LFS } & $\begin{array}{l}<2 \\
\mathrm{~dB} S P L\end{array}$ & TEOAEs & $\begin{array}{c}83 \% \\
76.54-88.99\end{array}$ & $\begin{array}{c}63 \% \\
54.05-71.94\end{array}$ & $\begin{array}{c}74 \% \\
66.85-80.52\end{array}$ & $\begin{array}{c}75 \% \\
65.67-83.3\end{array}$ & $\begin{array}{c}2.28 \\
1.78-2.91\end{array}$ & Small & $\begin{array}{c}0.26 \\
0.18-0.38\end{array}$ & Small \\
\hline & & DPOAES & $\begin{array}{c}89 \% \\
82.72-94.03\end{array}$ & $\begin{array}{c}65 \% \\
56.21-72.96\end{array}$ & $\begin{array}{c}71 \% \\
63.77-78.12\end{array}$ & $\begin{array}{c}86 \% \\
77.84-92.21\end{array}$ & $\begin{array}{c}2.55 \\
2.01-3.23\end{array}$ & Small & $\begin{array}{c}0.16 \\
0.10-0.27\end{array}$ & Moderate \\
\hline & $\begin{array}{l}<1 \\
\mathrm{~dB} S P L\end{array}$ & TEOAEs & $\begin{array}{c}51 \% \\
43.05-59.57\end{array}$ & $\begin{array}{c}90 \% \\
83.32-94.77\end{array}$ & $\begin{array}{c}87 \% \\
77.63-92.83\end{array}$ & $\begin{array}{c}60 \% \\
52.38-67.07\end{array}$ & $\begin{array}{c}5.18 \\
2.96-9.05\end{array}$ & Moderate & $\begin{array}{c}0.54 \\
0.45-0.64\end{array}$ & $\begin{array}{l}\text { Very } \\
\text { small }\end{array}$ \\
\hline & & DPOAEs & $\begin{array}{c}60 \% \\
50.99-67.75\end{array}$ & $\begin{array}{c}87 \% \\
79.89-92.44\end{array}$ & $\begin{array}{c}84 \% \\
75.32-90.57\end{array}$ & $\begin{array}{c}65 \% \\
57.67-72.67\end{array}$ & $\begin{array}{c}4.62 \\
2.87-7.44\end{array}$ & Small & $\begin{array}{c}0.46 \\
0.38-0.57\end{array}$ & Small \\
\hline \multirow[t]{4}{*}{ HFS } & $\begin{array}{l}<2 \\
\mathrm{~dB} S P L\end{array}$ & TEOAEs & $\begin{array}{c}88 \% \\
80.88-92.60\end{array}$ & $\begin{array}{c}51 \% \\
41.62-59.98\end{array}$ & $\begin{array}{c}67 \% \\
59.27-73.50\end{array}$ & $\begin{array}{c}78 \% \\
67.80-86.94\end{array}$ & $\begin{array}{c}1.78 \\
1.47-2.16\end{array}$ & $\begin{array}{l}\text { Very } \\
\text { small }\end{array}$ & $\begin{array}{c}0.24 \\
0.15-0.39\end{array}$ & Small \\
\hline & & DPOAES & $\begin{array}{c}97 \% \\
91.69-99.08\end{array}$ & $\begin{array}{c}50 \% \\
40.20-58.97\end{array}$ & $\begin{array}{c}66 \% \\
58.76-73.24\end{array}$ & $\begin{array}{c}94 \% \\
84.30-98.21\end{array}$ & $\begin{array}{c}1.92 \\
1.60-2.30\end{array}$ & $\begin{array}{l}\text { Very } \\
\text { small }\end{array}$ & $\begin{array}{c}0.07 \\
0.03-0.18\end{array}$ & Large \\
\hline & $\begin{array}{l}<1 \\
\mathrm{~dB} S P L\end{array}$ & TEOAEs & $\begin{array}{c}49 \% \\
40.27-57.58\end{array}$ & $\begin{array}{c}75 \% \\
66.54-82.60\end{array}$ & $\begin{array}{c}69 \% \\
58.88-78.07\end{array}$ & $\begin{array}{c}57 \% \\
48.49-64.31\end{array}$ & $\begin{array}{c}1.96 \\
1.38-2.81\end{array}$ & $\begin{array}{l}\text { Very } \\
\text { small }\end{array}$ & $\begin{array}{c}0.68 \\
0.56-0.82\end{array}$ & $\begin{array}{l}\text { Very } \\
\text { small }\end{array}$ \\
\hline & & DPOAES & $\begin{array}{c}78 \% \\
69.41-85.07\end{array}$ & $\begin{array}{c}74 \% \\
65.11-81.56\end{array}$ & $\begin{array}{c}75 \% \\
66.17-82.19\end{array}$ & $\begin{array}{c}77 \% \\
68.40-84.53\end{array}$ & $\begin{array}{c}2.99 \\
2.18-4.11\end{array}$ & Small & $\begin{array}{c}0.30 \\
0.21-0.43\end{array}$ & Small \\
\hline
\end{tabular}

OS = mean suppression for all frequencies, LFS = mean suppression for lower frequencies (1-2 kHz), HFS = mean suppression for higher frequencies (2.8-4 kHz for TEOAES and 2.8-6 kHz for DPOAEs), $P P V=$ positive predictive value, $N P V=$ negative predictive value, $L R=$ likelihood ratio. 
Table II. Results obtained among patients with either bilateral or no tinnitus.

\begin{tabular}{|c|c|c|c|c|c|c|c|c|c|c|}
\hline \multicolumn{3}{|c|}{$\begin{array}{l}\text { Statistical test } \\
\text { cut-off criterion }\end{array}$} & \multirow{2}{*}{$\begin{array}{c}\text { Sensitivity } \\
95 \% \mathrm{Cl} \\
86 \% \\
77.84-91.61\end{array}$} & \multirow{2}{*}{$\begin{array}{c}\text { Specificity } \\
95 \% \mathrm{Cl} \\
69 \% \\
58.02-78.69\end{array}$} & \multirow{2}{*}{$\begin{array}{c}\text { PPV } \\
95 \% \mathrm{Cl} \\
79 \% \\
70.35-85.58\end{array}$} & \multirow{2}{*}{$\begin{array}{c}\text { NPV } \\
95 \% \mathrm{Cl} \\
78 \% \\
67.28-87.11\end{array}$} & \multirow{2}{*}{$\begin{array}{l}\text { Positive LR } \\
95 \% \mathrm{Cl} \\
2.77 \\
1.99-3.85\end{array}$} & \multirow{2}{*}{$\begin{array}{l}\text { Diagnostic } \\
\text { value } \\
\text { Small }\end{array}$} & \multirow{2}{*}{$\begin{array}{c}\text { Negative LR } \\
95 \% \mathrm{Cl} \\
0.21 \\
0.13-0.33\end{array}$} & \multirow{2}{*}{$\begin{array}{c}\text { Diagnostic } \\
\text { value } \\
\text { Moderate }\end{array}$} \\
\hline OS & $\begin{array}{l}<2 \\
\mathrm{~dB} \text { SPL }\end{array}$ & TEOAEs & & & & & & & & \\
\hline & & DPOAES & $\begin{array}{c}96 \% \\
89.35-98.82\end{array}$ & $\begin{array}{c}65 \% \\
55.02-74.64\end{array}$ & $\begin{array}{c}72 \% \\
63.57-80.04\end{array}$ & $\begin{array}{c}94 \% \\
85.62-98.37\end{array}$ & $\begin{array}{c}2.76 \\
2.10-3.63\end{array}$ & Small & $\begin{array}{c}0.07 \\
0.02-0.17\end{array}$ & Large \\
\hline & $\begin{array}{l}<1 \\
\mathrm{~dB} S P L\end{array}$ & TEOAEs & $\begin{array}{c}51 \% \\
41.27-60.46\end{array}$ & $\begin{array}{c}95 \% \\
88.25-98.69\end{array}$ & $\begin{array}{c}93 \% \\
84.05-98.18\end{array}$ & $\begin{array}{c}59 \% \\
50.47-67.63\end{array}$ & $\begin{array}{c}10.69 \\
4.04-28.29\end{array}$ & Large & $\begin{array}{c}0.52 \\
0.42-0.63\end{array}$ & $\begin{array}{l}\text { Very } \\
\text { small }\end{array}$ \\
\hline & & DPOAES & $\begin{array}{c}73 \% \\
63.54-81.59\end{array}$ & $\begin{array}{c}90 \% \\
81.67-95.27\end{array}$ & $\begin{array}{c}89 \% \\
80.41-94.92\end{array}$ & $\begin{array}{c}75 \% \\
66.04-83.00\end{array}$ & $\begin{array}{c}7.41 \\
3.94-13.93\end{array}$ & Moderate & $\begin{array}{c}0.30 \\
0.21-0.41\end{array}$ & Small \\
\hline \multirow[t]{4}{*}{ LFS } & $\begin{array}{l}<2 \\
\mathrm{~dB} \text { SPL }\end{array}$ & TEOAEs & $\begin{array}{c}83 \% \\
74.78-89.47\end{array}$ & $\begin{array}{c}67 \% \\
56.30-77.35\end{array}$ & $\begin{array}{c}78 \% \\
68.98-84.62\end{array}$ & $\begin{array}{c}75 \% \\
63.30-84.01\end{array}$ & $\begin{array}{c}2.55 \\
1.85-3.52\end{array}$ & Small & $\begin{array}{c}0.25 \\
0.16-0.39\end{array}$ & Small \\
\hline & & DPOAES & $\begin{array}{c}91 \% \\
83.58-96.17\end{array}$ & $\begin{array}{c}69 \% \\
59.18-78.51\end{array}$ & $\begin{array}{c}74 \% \\
65.26-82.09\end{array}$ & $\begin{array}{c}89 \% \\
79.80-95.22\end{array}$ & $\begin{array}{c}2.99 \\
2.19-4.08\end{array}$ & Small & $\begin{array}{c}0.13 \\
0.06-0.25\end{array}$ & Moderate \\
\hline & $\begin{array}{l}<1 \\
\mathrm{~dB} \text { SPL }\end{array}$ & TEOAES & $\begin{array}{c}45 \% \\
35.24-54.33\end{array}$ & $\begin{array}{c}94 \% \\
86.50-98.02\end{array}$ & $\begin{array}{c}91 \% \\
80.05-96.98\end{array}$ & $\begin{array}{c}56 \% \\
47.08-64.10\end{array}$ & $\begin{array}{c}7.41 \\
3.09-17.77\end{array}$ & Moderate & $\begin{array}{c}0.59 \\
0.49-0.70\end{array}$ & $\begin{array}{l}\text { Very } \\
\text { small }\end{array}$ \\
\hline & & DPOAES & $\begin{array}{c}62 \% \\
51.61-71.21\end{array}$ & $\begin{array}{c}89 \% \\
80.85-95.04\end{array}$ & $\begin{array}{c}88 \% \\
77.59-94.12\end{array}$ & $\begin{array}{c}66 \% \\
56.67-74.65\end{array}$ & $\begin{array}{c}5.83 \\
3.09-11.02\end{array}$ & Moderate & $\begin{array}{c}0.43 \\
0.33-0.55\end{array}$ & Small \\
\hline \multirow[t]{4}{*}{ HFS } & $\begin{array}{l}<2 \\
\mathrm{~dB} S P L\end{array}$ & TEOAEs & $\begin{array}{c}85 \% \\
76.01-91.17\end{array}$ & $\begin{array}{c}51 \% \\
39.40-61.76\end{array}$ & $\begin{array}{c}67 \% \\
57.92-75.12\end{array}$ & $\begin{array}{c}74 \% \\
60.34-84.46\end{array}$ & $\begin{array}{c}1.71 \\
1.36-2.17\end{array}$ & $\begin{array}{l}\text { Very } \\
\text { small }\end{array}$ & $\begin{array}{c}0.30 \\
0.18-0.50\end{array}$ & Small \\
\hline & & DPOAES & $\begin{array}{c}98 \% \\
91.47-99.70\end{array}$ & $\begin{array}{c}49 \% \\
37.23-60.31\end{array}$ & $\begin{array}{c}67 \% \\
57.48-75.01\end{array}$ & $\begin{array}{c}95 \% \\
83.08-99.39\end{array}$ & $\begin{array}{c}1.90 \\
1.53-2.37\end{array}$ & $\begin{array}{l}\text { Very } \\
\text { small }\end{array}$ & $\begin{array}{c}0.05 \\
0.01-0.20\end{array}$ & Large \\
\hline & $\begin{array}{l}<1 \\
\mathrm{~dB} S P L\end{array}$ & TEOAEs & $\begin{array}{c}47 \% \\
36.78-57.29\end{array}$ & $\begin{array}{c}78 \% \\
67.54-86.44\end{array}$ & $\begin{array}{c}72 \% \\
59.24-82.40\end{array}$ & $\begin{array}{c}55 \% \\
45.66-64.41\end{array}$ & $\begin{array}{c}2.14 \\
1.35-3.38\end{array}$ & Small & $\begin{array}{c}0.68 \\
0.55-0.85\end{array}$ & $\begin{array}{l}\text { Very } \\
\text { small }\end{array}$ \\
\hline & & DPOAES & $\begin{array}{c}80 \% \\
69.56-88.11\end{array}$ & $\begin{array}{c}76 \% \\
65.42-85.05\end{array}$ & $\begin{array}{c}77 \% \\
66.58-85.62\end{array}$ & $\begin{array}{c}79 \% \\
68.46-87.63\end{array}$ & $\begin{array}{c}3.37 \\
2.24-5.06\end{array}$ & Small & $\begin{array}{c}0.26 \\
0.17-0.41\end{array}$ & Small \\
\hline
\end{tabular}

OS = mean suppression for all frequencies, LFS = mean suppression for lower frequencies (1-2 kHz), HFS = mean suppression for higher frequencies (2.8-4 kHz for TEOAES and 2.8-6 kHz for DPOAES), $L R=$ llikelihood ratio.

are probably the reasons behind the lack of effective and aetiological treatment options. The involvement of the medial olivocochlear bundle (MOCB) in tinnitus generation has been hypothesised in the following concepts. A reduction in neural efferent control of the cochlear amplifier may result in an increase of its gain and enhancement of spontaneous activity in the auditory nerve or other plastic readjustments in the central auditory system ${ }^{15}$. Owing to the diffuse efferent innervation of outer hair cells (one fibre for 20-30 outer hair cells), efferent nerve fibres with reduced afferent input is shared with neighbouring outer hair cells partnering undamaged inner hair cells. As a result, those inner hair cells may receive defective efferent inhibition and thus create an area of hyperactivity in the basilar membrane that may be perceived as tinnitus ${ }^{16}$. Furthermore, the perceived tinnitus intensity and associated annoyance might be influenced by the efferent system through its connections with the reticular formation within the brainstem ${ }^{17}$. Furthermore, cochlear efferent innervation seems to be able to suppress the increased spontaneous firing rate in the inferior colliculus of a guinea pig model, where tinnitus was developed through acoustic trauma ${ }^{18}$.
Conduction of separate statistical analyses for lower and higher frequencies was based on the assumption that with patients reporting high frequency tinnitus, different results might arise for lower and higher frequency bands (Table I). However, this hypothesis was not confirmed. At high frequencies, the dysfunction of TEOAEs suppression seemed to loose its relationship with tinnitus. This may be partly due to the fact that recordings at high frequencies were absent in a large number of ears. On the other hand, DPOAEs suppression values lower than $2 \mathrm{~dB}$ SPL maintained a negative predictive value of $94 \%$, which means that there was a high probability and that tinnitus was not present when DPOAEs suppression values were larger than $2 \mathrm{~dB}$ SPL at high frequencies (test negative). The absence of measurements in a large number of ears, which is also true for DPOAEs at high frequencies, is expected to have compromised the results of our analysis. At low frequencies, defective TEOAEs suppression $(<1 \mathrm{~dB}$ SPL) maintained its association with the presence of tinnitus, which may reflect the fact that TEOAEs actually render information that refers to outer hair cells $(\mathrm{OHC})$ and MOCB functionality through the entire cochlea ${ }^{19-22}$. DPOAEs suppression values lower than $2 \mathrm{~dB}$ SPL 
Table III. Results obtained for the cut-off criteria used in this study for patients according to age.

\begin{tabular}{|c|c|c|c|c|c|c|c|c|c|c|}
\hline \multicolumn{3}{|c|}{$\begin{array}{l}\text { Statistical test } \\
\text { cut-off criterion }\end{array}$} & \multirow{2}{*}{$\begin{array}{c}\text { Sensitivity } \\
95 \% \mathrm{Cl} \\
88 \% \\
67.64-97.34\end{array}$} & \multirow{2}{*}{$\begin{array}{c}\text { Specificity } \\
95 \% \mathrm{Cl} \\
70 \% \\
50.60-85.27\end{array}$} & \multirow{2}{*}{$\begin{array}{c}\text { PPV } \\
95 \% \mathrm{Cl} \\
70 \% \\
50.60-85.27\end{array}$} & \multirow{2}{*}{$\begin{array}{c}\text { NPV } \\
95 \% \mathrm{Cl} \\
88 \% \\
67.64-97.34\end{array}$} & \multirow{2}{*}{$\begin{array}{c}\text { Positive LR } \\
95 \% \mathrm{Cl} \\
2.92 \\
1.65-5.14\end{array}$} & \multirow{2}{*}{$\begin{array}{c}\text { Diagnostic } \\
\text { value } \\
\text { Small }\end{array}$} & \multirow{2}{*}{$\begin{array}{c}\text { Negative LR } \\
95 \% \mathrm{Cl} \\
0.18 \\
0.06-0.53\end{array}$} & \multirow{2}{*}{$\begin{array}{l}\begin{array}{c}\text { Diagnostic } \\
\text { value }\end{array} \\
\text { Moderate }\end{array}$} \\
\hline $\begin{array}{l}20-35 \\
\text { years }\end{array}$ & $\begin{array}{l}<2 \\
\mathrm{~dB} S P L\end{array}$ & TEOAEs & & & & & & & & \\
\hline & & DPOAES & $\begin{array}{c}92 \% \\
73-98.97\end{array}$ & $\begin{array}{c}60 \% \\
40.60-77.34\end{array}$ & $\begin{array}{c}65 \% \\
46.49-80.25\end{array}$ & $\begin{array}{c}90 \% \\
68.30-98.77\end{array}$ & $\begin{array}{c}2.29 \\
1.45-3.61\end{array}$ & Small & $\begin{array}{c}0.14 \\
0.04-0.54\end{array}$ & Moderate \\
\hline & $\begin{array}{l}<1 \\
\mathrm{~dB} \mathrm{SPL}\end{array}$ & TEOAES & $\begin{array}{c}58 \% \\
36.64-77.89\end{array}$ & $\begin{array}{c}97 \% \\
82.78-99.92\end{array}$ & $\begin{array}{c}93 \% \\
68.05-99.83\end{array}$ & $\begin{array}{c}74 \% \\
57.87-86.96\end{array}$ & $\begin{array}{c}17.50 \\
2.47-123.80\end{array}$ & Large & $\begin{array}{c}0.43 \\
0.27-0.7\end{array}$ & Small \\
\hline & & DPOAES & $\begin{array}{c}79 \% \\
57.85-92.87\end{array}$ & $\begin{array}{c}93 \% \\
77.93-99.18\end{array}$ & $\begin{array}{c}90 \% \\
69.62-98.83\end{array}$ & $\begin{array}{c}85 \% \\
68.10-94.89\end{array}$ & $\begin{array}{c}11.88 \\
3.06-46.02\end{array}$ & Large & $\begin{array}{c}0.22 \\
0.10-0.49\end{array}$ & Small \\
\hline \multirow[t]{4}{*}{$\begin{array}{l}36-50 \\
\text { years }\end{array}$} & $\begin{array}{l}<2 \\
\mathrm{~dB} \mathrm{SPL}\end{array}$ & TEOAEs & $\begin{array}{c}93 \% \\
79.61-98.43\end{array}$ & $\begin{array}{c}54 \% \\
32.82-74.45\end{array}$ & $\begin{array}{c}77 \% \\
62.69-87.97\end{array}$ & $\begin{array}{c}81 \% \\
54.35-95.95\end{array}$ & $\begin{array}{c}2.02 \\
1.29-3.15\end{array}$ & Small & $\begin{array}{c}0.14 \\
0.04-0.44\end{array}$ & Moderate \\
\hline & & DPOAES & $\begin{array}{c}89 \% \\
75.20-97.06\end{array}$ & $\begin{array}{c}65 \% \\
44.33-82.79\end{array}$ & $\begin{array}{c}79 \% \\
63.96-89.96\end{array}$ & $\begin{array}{c}81 \% \\
58.09-94.55\end{array}$ & $\begin{array}{c}2.58 \\
1.51-4.43\end{array}$ & Small & $\begin{array}{c}0.16 \\
0.06-0.42\end{array}$ & Moderate \\
\hline & $\begin{array}{l}<1 \\
\mathrm{~dB} S \mathrm{SPL}\end{array}$ & TEOAES & $\begin{array}{c}53 \% \\
36.13-68.49\end{array}$ & $\begin{array}{c}88 \% \\
67.64-97.34\end{array}$ & $\begin{array}{c}88 \% \\
67.64-97.34\end{array}$ & $\begin{array}{c}53 \% \\
36.13-68.49\end{array}$ & $\begin{array}{c}4.20 \\
1.40-12.60\end{array}$ & Small & $\begin{array}{c}0.54 \\
0.38-0.78\end{array}$ & $\begin{array}{l}\text { Very } \\
\text { small }\end{array}$ \\
\hline & & DPOAES & $\begin{array}{c}77 \% \\
60.67-88.87\end{array}$ & $\begin{array}{c}92 \% \\
73.97-99.02\end{array}$ & $\begin{array}{c}94 \% \\
79.19-99.23\end{array}$ & $\begin{array}{c}72 \% \\
53.25-86.25\end{array}$ & $\begin{array}{c}9.62 \\
2.52-36.74\end{array}$ & Moderate & $\begin{array}{c}0.25 \\
0.14-0.45\end{array}$ & Small \\
\hline \multirow[t]{4}{*}{$\begin{array}{l}51-60 \\
\text { years }\end{array}$} & $\begin{array}{l}<2 \\
\mathrm{~dB} \mathrm{SPL}\end{array}$ & TEOAEs & $\begin{array}{c}88 \% \\
76.56-95.65\end{array}$ & $\begin{array}{c}76 \% \\
62.36-86.51\end{array}$ & $\begin{array}{c}78 \% \\
65.27-87.71\end{array}$ & $\begin{array}{c}87 \% \\
74.26-95.17\end{array}$ & $\begin{array}{c}3.67 \\
2.27-5.96\end{array}$ & Small & $\begin{array}{c}0.15 \\
0.07-0.33\end{array}$ & Moderate \\
\hline & & DPOAES & $\begin{array}{c}100 \% \\
92.89-100\end{array}$ & $\begin{array}{c}68 \% \\
54.76-80.09\end{array}$ & $\begin{array}{c}74 \% \\
61.43-83.50\end{array}$ & $100 \%$ & $\begin{array}{c}3.17 \\
2.16-4.64\end{array}$ & Small & 0 & Large \\
\hline & $\begin{array}{l}<1 \\
\mathrm{~dB} S \mathrm{SPL}\end{array}$ & TEOAES & $\begin{array}{c}65 \% \\
50.91-78.03\end{array}$ & $\begin{array}{c}87 \% \\
75.10-94.63\end{array}$ & $\begin{array}{c}83 \% \\
67.94-92.85\end{array}$ & $\begin{array}{c}72 \% \\
59.81-82.69\end{array}$ & $\begin{array}{c}5.04 \\
2.46-10.35\end{array}$ & Moderate & $\begin{array}{c}0.40 \\
0.27-0.59\end{array}$ & Small \\
\hline & & DPOAES & $\begin{array}{c}76 \% \\
61.13-86.66\end{array}$ & $\begin{array}{c}89 \% \\
78.48-96.04\end{array}$ & $\begin{array}{c}86 \% \\
72.07-94.7\end{array}$ & $\begin{array}{c}81 \% \\
69.09-89.75\end{array}$ & $\begin{array}{c}7.17 \\
3.31-15.55\end{array}$ & Moderate & $\begin{array}{c}0.27 \\
0.17-0.45\end{array}$ & Small \\
\hline \multirow[t]{4}{*}{$\begin{array}{l}>61 \\
\text { years }\end{array}$} & $\begin{array}{l}<2 \\
d B S P L\end{array}$ & TEOAEs & $\begin{array}{c}80 \% \\
63.06-91.56\end{array}$ & $\begin{array}{c}53 \% \\
26.59-78.73\end{array}$ & $\begin{array}{c}80 \% \\
63.06-91.56\end{array}$ & $\begin{array}{c}53 \% \\
26.59-78.73\end{array}$ & $\begin{array}{c}1.71 \\
0.97-3.02\end{array}$ & $\begin{array}{l}\text { Very } \\
\text { small }\end{array}$ & $\begin{array}{c}0.37 \\
0.17-0.85\end{array}$ & Small \\
\hline & & DPOAES & $\begin{array}{c}95 \% \\
75.13-99.87\end{array}$ & $\begin{array}{c}65 \% \\
46.49-80.25\end{array}$ & $\begin{array}{c}61 \% \\
42.19-78.15\end{array}$ & $\begin{array}{c}96 \% \\
78.05-99.89\end{array}$ & $\begin{array}{c}2.69 \\
1.69-4.29\end{array}$ & Small & $\begin{array}{c}0.08 \\
0.03-0.23\end{array}$ & Large \\
\hline & $\begin{array}{l}<1 \\
\mathrm{~dB} S P L\end{array}$ & TEOAES & $\begin{array}{c}34 \% \\
19.13-52.21\end{array}$ & $\begin{array}{c}100 \% \\
78.20-100\end{array}$ & $\begin{array}{c}100 \% \\
73.54-100\end{array}$ & $\begin{array}{c}39 \% \\
24.04-56.61\end{array}$ & $\begin{array}{c}\text { Not } \\
\text { applicable }\end{array}$ & $\begin{array}{c}\text { Not } \\
\text { applicable }\end{array}$ & $\begin{array}{c}0.66 \\
0.52-0.83\end{array}$ & $\begin{array}{l}\text { Very } \\
\text { small }\end{array}$ \\
\hline & & DPOAES & $\begin{array}{c}68 \% \\
47.65-84.12\end{array}$ & $\begin{array}{c}67 \% \\
40.99-86.66\end{array}$ & $\begin{array}{c}76 \% \\
54.87-90.64\end{array}$ & $\begin{array}{c}57 \% \\
34.02-78.18\end{array}$ & $\begin{array}{c}2.04 \\
1.01-4.10\end{array}$ & Small & $\begin{array}{c}0.48 \\
0.26-0.90\end{array}$ & Small \\
\hline
\end{tabular}

$O S=$ mean suppression for all frequencies, $P P V=$ positive predictive value, $N P V=$ negative predictive value, $L R=$ likelihood ratio, $C l=$ confidence interval.

maintained a high negative predictive value. Mean suppression values (for all frequencies) were demonstrated to be of higher diagnostic value than suppression values corresponding to either lower or higher frequencies (Tables I, II).

Negative or positive associations were stronger among patients with either bilateral or no tinnitus than among patients with unilateral tinnitus (Table II). This probably confirms the observation that defective function of the medial olivocochlear bundle usually applies to the contralateral ear as well, with lateralisation of tinnitus in these cases possibly originating from central nervous system mechanisms ${ }^{48}$. Functional magnetic resonance imaging studies have also demonstrated that brain hyperactivity in tinnitus patients may not be lateralised, despite the unilateral perception of the symptom ${ }^{23}$. Mean suppression values (for all frequencies) were better predictors for the presence or absence of tinnitus than suppression values corresponding to either lower or higher frequencies (Table I).

Patients with diverse age groups were included so as to investigate the applicability of the selected cut-off points in clinical practice. Regarding age, analysis of the results by age-group in Table III verified that age is not a trivial confounding factor, since age groups 20-35, 36-50 and 51-60 years seem to present similar results to each other and to the general population. Despite the subdivision of the population into smaller groups, the selected cut-off points consistently retained their predictive abilities, both regarding the high probability of a negative test result given the absence of tinnitus in case of OS $<2 \mathrm{~dB}$ SPL and regarding the high probability of a positive test result in the absence of tinnitus for OS $<1 \mathrm{~dB}$ SPL. With respect to the group of patients older than 61 years, the results were less clear, but this may 
Table IV. Results obtained by gender.

\begin{tabular}{|c|c|c|c|c|c|c|c|c|c|c|}
\hline \multicolumn{2}{|c|}{$\begin{array}{l}\text { Statistical test } \\
\text { cut-off criterion }\end{array}$} & & $\begin{array}{l}\text { Sensitivity } \\
95 \% \mathrm{Cl}\end{array}$ & $\begin{array}{l}\text { Specificity } \\
95 \% \mathrm{Cl}\end{array}$ & $\begin{array}{c}\text { PPV } \\
95 \% \mathrm{Cl}\end{array}$ & $\begin{array}{l}\text { NPV } \\
95 \% \mathrm{Cl}\end{array}$ & $\begin{array}{c}\text { Positive LR } \\
95 \% \mathrm{Cl}\end{array}$ & $\begin{array}{l}\text { Diagnostic } \\
\text { value }\end{array}$ & $\begin{array}{c}\text { Negative LR } \\
95 \% \mathrm{Cl}\end{array}$ & $\begin{array}{l}\text { Diagnostic } \\
\text { value }\end{array}$ \\
\hline \multirow[t]{4}{*}{ Female } & $\begin{array}{l}\mathrm{OS}<2 \\
\mathrm{~dB} S P L\end{array}$ & TEOAEs & $\begin{array}{c}84 \% \\
74.42-91.28\end{array}$ & $\begin{array}{c}67 \% \\
54.57-77.34\end{array}$ & $\begin{array}{c}74 \% \\
64.08-82.71\end{array}$ & $\begin{array}{c}79 \% \\
66.32-88.14\end{array}$ & $\begin{array}{c}2.52 \\
1.80-3.55\end{array}$ & Small & $\begin{array}{c}0.24 \\
0.14-0.40\end{array}$ & Small \\
\hline & & DPOAES & $\begin{array}{c}93 \% \\
85.12-97.80\end{array}$ & $\begin{array}{c}54 \% \\
42.83-65.69\end{array}$ & $\begin{array}{c}66 \% \\
56.20-74.96\end{array}$ & $\begin{array}{c}90 \% \\
77.34-96.53\end{array}$ & $\begin{array}{c}2.05 \\
1.60-2.63\end{array}$ & Small & $\begin{array}{c}0.12 \\
0.05-0.29\end{array}$ & Moderate \\
\hline & $\begin{array}{l}O S<1 \\
d B S P L\end{array}$ & TEOAEs & $\begin{array}{c}54 \% \\
42.30-64.75\end{array}$ & $\begin{array}{c}94 \% \\
86.38-98.47\end{array}$ & $\begin{array}{c}92 \% \\
80.02-97.68\end{array}$ & $\begin{array}{c}64 \% \\
54.26-73.23\end{array}$ & $\begin{array}{c}9.66 \\
3.65-25.57\end{array}$ & Moderate & $\begin{array}{c}0.49 \\
0.39-0.62\end{array}$ & Small \\
\hline & & DPOAES & $\begin{array}{c}74 \% \\
63.21-83.58\end{array}$ & $\begin{array}{c}91 \% \\
81.71-96.16\end{array}$ & $\begin{array}{c}89 \% \\
79.06-95.56\end{array}$ & $\begin{array}{c}77 \% \\
67.11-85.53\end{array}$ & $\begin{array}{c}7.97 \\
3.89-16.32\end{array}$ & Moderate & $\begin{array}{c}0.28 \\
0.19-0.42\end{array}$ & Small \\
\hline \multirow[t]{4}{*}{ Male } & $\begin{array}{l}\mathrm{OS}<2 \\
\mathrm{~dB} \mathrm{SPL}\end{array}$ & TEOAES & $\begin{array}{c}91 \% \\
82.03-96.74\end{array}$ & $\begin{array}{c}69 \% \\
54.11-80.89\end{array}$ & $\begin{array}{c}80 \% \\
69.20-87.96\end{array}$ & $\begin{array}{c}85 \% \\
70.83-94.43\end{array}$ & $\begin{array}{c}2.91 \\
1.93-4.40\end{array}$ & Small & $\begin{array}{c}0.13 \\
0.06-0.28\end{array}$ & Moderate \\
\hline & & DPOAES & $\begin{array}{c}95 \% \\
85.38-98.90\end{array}$ & $\begin{array}{c}48 \% \\
35.23-61.61\end{array}$ & $\begin{array}{c}64 \% \\
52.38-73.71\end{array}$ & $\begin{array}{c}91 \% \\
74.98-98.02\end{array}$ & $\begin{array}{c}1.83 \\
1.42-2.36\end{array}$ & $\begin{array}{l}\text { Very } \\
\text { small }\end{array}$ & $\begin{array}{c}0.11 \\
0.04-0.34\end{array}$ & Moderate \\
\hline & $\begin{array}{l}0 S<1 \\
\mathrm{~dB} S P L\end{array}$ & TEOAEs & $\begin{array}{c}54 \% \\
41.20-65.72\end{array}$ & $\begin{array}{c}86 \% \\
73.74-94.30\end{array}$ & $\begin{array}{c}84 \% \\
69.93-93.36\end{array}$ & $\begin{array}{c}58 \% \\
46.02-69.14\end{array}$ & $\begin{array}{c}3.91 \\
1.90-8.04\end{array}$ & Small & $\begin{array}{c}0.54 \\
0.41-0.71\end{array}$ & $\begin{array}{l}\text { Very } \\
\text { small }\end{array}$ \\
\hline & & DPOAES & $\begin{array}{c}76 \% \\
63.26-85.78\end{array}$ & $\begin{array}{c}84 \% \\
71.20-92.23\end{array}$ & $\begin{array}{c}84 \% \\
71.67-92.38\end{array}$ & $\begin{array}{c}75 \% \\
62.71-85.54\end{array}$ & $\begin{array}{c}4.63 \\
2.51-8.56\end{array}$ & Small & $\begin{array}{c}0.29 \\
0.18-0.46\end{array}$ & Small \\
\hline
\end{tabular}

OS = mean suppression values for all frequencies, $L R=$ likelihood ratio.

be partly attributed to the fact that in this group of patients OAEs may not be recorded in multiple frequencies, possibly due to a subclinical presbycusis, thus compromising statistical calculations.

An unexpected finding of this study was the difference between genders (Table IV). In females $(n=78)$, the association between objective findings and subjective tinnitus seem to refer mainly to the presence of disease, with DPOAEs only reaching the criteria of a useful correlation between the possible absence of tinnitus and the recording of negative results. In males $(n=60)$, however, powerful correlations were demonstrated only for the absence of the disease in patients with mean suppression values larger than $2 \mathrm{~dB}$ SPL. The limited number of patients included in each subpopulation may account for the differences in results. Emotional and attentional state, psychological disorders, hyperacousis or temporo-mandibular joint lesions are some additional known causes involved in the generation, development and perception of tinnitus ${ }^{24-27}$. The underlying mechanisms remain obscure and seem to regard both central and peripheral auditory pathway structures interacting with or projecting to multiple non-auditory central nervous system structures. The patients included in the study were questioned regarding medical history of psychological or mood disorders. The study did not include any further investigation, for example through suitable questionnaires. Patients were not queried regarding possible temporo-mandibular joint disorders or hyperacousis. These might be considered as limitations of this study. Further studies including larger populations divided into subgroups according to the aforementioned possible tinnitus triggers may reveal additional information on the pathophysiology of tinnitus.

\section{Conclusions}

The results of this study appear to reinforce the hypothesis that defective MOCB function may play a crucial role in the pathogenesis of tinnitus in normal hearing patients. Mean suppression values were demonstrated to be potentially useful objective tools in prediction of the presence or absence of subjective tinnitus. Mean suppression values (for all frequencies) were demonstrated to have stronger associations with tinnitus presence/absence than suppression values referring to either lower of higher frequencies. Gender and tinnitus laterality appear to be important confounding factors that should be taken into consideration. The potential clinical implications of this observation seem to extend to a broad age range (20-60 years old) with young tinnitus patients $(<35$ years old) exhibiting strong and clear associations, while older patients ( $>61$ years old) presented inconclusive results. OAEs suppression seems to be a useful tool that could provide insight into a subjective symptom of normal hearing subjects of diverse age groups.

\section{References}

1 Chéry-Croze S, Collet L, Morgon A. Medial olivo-cochlear system and tinnitus. Acta Otolaryngol 1993;113:285-90.

2 Attias J, Bresloff I, Furman V. The influence of the efferent auditory system on otoacoustic emissions in noise induced tinnitus: clinical relevance. Acta Otolaryngol 1996;116:534-9. 
3 Ceranic BJ, Prasher DK, Raglan E, et al. Tinnitus after head injury: evidence from otoacoustic emissions. J Neurol Neurosurg Psychiatry 1998;65:523-9.

4 Fávero ML, Sanchez TG, Bento RF, et al. Contralateral suppression of otoacoustic emission in patients with tinnitus. Braz J Otorhinolaryngol 2006;72:223-6.

5 Fernandes Lda C, Santos TM. Tinnitus and normal hearing: a study on the transient otoacoustic emissions suppression. Braz J Otorhinolaryngol 2009;75:414-9.

6 Komis A, Maragkoudakis P, Gkoritsa E, et al. The effect of tinnitus and presbycusis on contralateral suppression of otoacoustic emissions. JHS 2014;4:9-20.

7 Lalaki P, Hatzopoulos S, Lorito G, et al. A connection between the efferent auditory system and noise-induced tinnitus generation. Reduced contralateral suppression of TEOAEs in patients with noise-induced tinnitus. Med Sci Monit 2011;17:MT56-62.

8 Riga M, Papadas T, Werner JA, et al. A clinical study of the efferent auditory system in patients with normal hearing who have acute tinnitus. Otol Neurotol 2007;28:185-90.

9 Geven LI, Wit HP, de Kleine E, et al. Wavelet analysis demonstrates no abnormality in contralateral suppression of otoacoustic emissions in tinnitus patients. Hear Res 2012;286:30-40.

10 Paglialonga A, Fiocchi S, Del Bo L, et al. Quantitative analysis of cochlear active mechanisms in tinnitus subjects with normal hearing sensitivity: time-frequency analysis of transient evoked otoacoustic emissions and contralateral suppression. Auris Nasus Larynx 2011;38:33-40.

11 Riga M, Katotomichelakis M, Danielides V. The potential role of the medial olivocochlear bundle in the generation of tinnitus: controversies and weaknesses in the existing clinical studies. Otol Neurotol 2015;36:201-8.

12 de Azevedo AA, Langguth B, de Oliveira PM, et al. Tinnitus treatment with piribedil guided by electrocochleography and acoustic otoemissions. Otol Neurotol 2009;30:676-80.

13 Bidelman GM, Bhagat SP. Right-ear advantage drives the link between olivocochlear efferent 'antimasking' and speech-innoise listening benefits. Neuroreport 2015;26:483-7.

14 Riga M, Komis A, Maragkoudakis P, et al. Objective assessment of subjective tinnitus through contralateral suppression of otoacoustic emissions by white noise; suggested cut-off points. Int J Audiol 2016;55:775-81.

15 Weisz N, Hartmann T, Dohrmann K, et al. High-frequency tinnitus without hearing loss does not mean absence of deafferentation. Hear Res 2006;222:108-14.

16 Chery-Croze S, Truy E, Morgon A. Contralateral suppression of transiently evoked otoacoustic emissions and tinnitus. Br J Audiol 1994;28:255-66.

17 Hazell JW, Jastreboff PJ. Tinnitus I: Auditory mechanisms: a model for tinnitus and hearing impairment. J Otolaryngol 1990;19:1-5.

18 Mulders WH, Seluakumaran K, Robertson D. Efferent pathways modulate hyperactivity in inferior colliculus. J Neurosci 2010;30:9578-87.

19 Probst R, Harris FP. Transiently evoked and distortion product otoacoustic emissions. Comparison of results from normally hearing and hearing impaired human ears. Arch Otolaryngol Head Neck Surg 1993;119:858-60.

20 Wagner W, Plinkert PK. The relation between audiology threshold and evoked otoacoustic emissions. Eur Arch Otorhinolaryngol 1999;256:177-88.

21 Granjeiro RC, Kehrle HM, Bezerra RL, et al. Transient and distortion product evoked oto-acoustic emissions in normal hearing patients with and without tinnitus. Otolaryngol Head Neck Surg 2008;138:502-6.

22 Henry JA, Roberts LE, Caspary DM, et al. Underlying mechanisms of tinnitus: review and clinical implications. J Am Acad Audiol 2014;25:5-22.

23 Shore SE, Roberts LE, Langguth B. Maladaptive plasticity in tinnitus--triggers, mechanisms and treatment. Nat Rev Neurol 2016;12:150-60.

24 Salviati M, Bersani FS, Valeriani G, et al. A brain centred view of psychiatric comorbidity in tinnitus: from otology to hodology. Neural Plast 2014;2014:817852.

25 Cianfrone G, Mazzei F, Salviati M, et al. Tinnitus Holistic Simplified Classification (THoSC): a new assessment for subjective tinnitus, with diagnostic and therapeutic implications. Ann Otol Rhinol Laryngol 2015;124:550-60.

26 Eggermont JJ. The auditory cortex and tinnitus - a review of animal and human studies. Eur J Neurosci 2015;41:665-76.

Received: October 14, 2016 - Accepted: May 7, 2017

Address for correspondence: Maria Riga, Democritus University of Thrace, PO Box 2206, Palagia, 68100, Alexandroupolis, Greece. Tel. +30 6977325466. Fax +30 25510 39986. E-mail: mariariga@ hotmail.com 